Article

\title{
Estrada Index of Random Bipartite Graphs
}

\section{Yilun Shang}

Department of Mathematics, Tongji University, Shanghai 200092, China; E-Mail: shyl@tongji.edu.cn Academic Editor: Sergei D. Odintsov

Received: 17 October 2015 / Accepted: 30 November 2015 / Published: 7 December 2015

\begin{abstract}
The Estrada index of a graph $G$ of $n$ vertices is defined by $E E(G)=\sum_{i=1}^{n} e^{\lambda_{i}}$, where $\lambda_{1}, \lambda_{2}, \cdots, \lambda_{n}$ are the eigenvalues of $G$. In this paper, we give upper and lower bounds of $E E(G)$ for almost all bipartite graphs by investigating the upper and lower bounds of the spectrum of random matrices. We also formulate an exact estimate of $E E(G)$ for almost all balanced bipartite graphs.
\end{abstract}

Keywords: Estrada index; random graph; eigenvalues; limit spectral distribution

\section{Introduction}

The topological structures of many social, biological, and technological systems can be characterized by the connectivity properties of the interaction pathways (edges) between system components (vertices) [1]. Starting with the Königsberg seven-bridge problem in 1736, graphs with bidirectional or symmetric edges have ideally epitomized structures of various complex systems, and have developed into one of the mainstays of the modern discrete mathematics and network theory. Formally, a simple graph $G$ consists of a vertex set $V=\{1,2, \cdots, n\}$ and an edge set $E \subseteq V \times V$. The adjacency matrix of $G$ is a symmetric $(0,1)$-matrix $A(G)=\left(a_{i j}\right) \in \mathbb{R}^{n \times n}$, where $a_{i j}=a_{j i}=1$ if vertices $i$ and $j$ are adjacent, and $a_{i j}=a_{j i}=0$ otherwise. It is well-known in algebraic graph theory that $A(G)$ has exactly $n$ real eigenvalues $\lambda_{1} \geq \lambda_{2} \geq \cdots \geq \lambda_{n}$ due to its symmetry. They are usually called the spectrum (eigenvalues) of $G$ itself [2].

A spectral graph invariant, the Estrada index $E E(G)$ of $G$, is defined as

$$
E E(G)=\sum_{i=1}^{n} e^{\lambda_{i}}
$$

This quantity was introduced by Estrada [3] in 2000. It has noteworthy chemical applications, such as quantifying the degree of folding of long-chain molecules and the Shannon entropy [3-7]. The Estrada 
index provides a remarkable measure of subgraph centrality as well as fault tolerance in the study of complex networks $[1,8-10]$. Building upon varied symmetric features in graphs, mathematical properties of this invariant can be found in, e.g., [11-18].

The Estrada index can be readily calculated once the eigenvalues are known. However, it is notoriously difficult to compute the eigenvalues of a large matrix even for $(0,1)$-matrix $A(G)$. In the past few years, researchers managed to establish a number of lower and upper bounds to estimate this invariant (see [13] for an updated survey). A common drawback is that only few classes of graphs attain the equalities of those bounds. Therefore, one may naturally wonder the typical behavior of the invariant $E E(G)$ for most graphs with respect to other graph parameters such as the number of vertices $n$.

The classical Erdős-Rényi random graph model $\mathcal{G}_{n}(p)$ includes the edges between all pairs of vertices independently at random with probability $p$ [19]. It has symmetric, bell-shaped degree distribution, which is shared by many other random graph models. Regarding the Estrada index, Chen et al. [20] showed the following result: Let $G_{n}(p) \in \mathcal{G}_{n}(p)$ be a random graph with a constant $p \in(0,1)$, then

$$
E E\left(G_{n}(p)\right)=e^{n p}\left(e^{O(\sqrt{n})}+o(1)\right) \quad \text { a.s. }
$$

Here, we say that a certain property $\mathcal{P}$ holds in $\mathcal{G}_{n}(p)$ almost surely (a.s.) if the probability that a random graph $G_{n}(p)$ has the property $\mathcal{P}$ converges to 1 as $n$ tends to infinity. Therefore, the result (1) presents an analytical estimate of the Estrada index for almost all graphs.

Our motivation in this paper is to investigate the Estrada index of random bipartite graphs, which is a natural bipartite version of Erdôs-Rényi random graphs. Bipartite graphs appear in a range of applications in timetabling, communication networks and computer science, where components of the systems are endowed with two different attributes and symmetric relations are only established between these two parts [21-23]. Formally, a bipartite graph is a graph whose vertices can be divided into two disjoint sets $V_{1}$ and $V_{2}$ such that every edge connects a vertex in $V_{1}$ to a vertex in $V_{2}$. A bipartite graph is a graph that does not contain any odd-length cycles; (chemical) trees are bipartite graphs. The random bipartite graph model is denoted by $\mathcal{G}_{n_{1}, n_{2}}(p)$, where $n_{i}=\left|V_{i}\right|$ for $i=1,2$, satisfying $n_{1}+n_{2}=n$.

The authors in [20] posed the following conjecture pertaining to bipartite graphs.

Conjecture 1. Let $G_{n_{1}, n_{2}}(p) \in \mathcal{G}_{n_{1}, n_{2}}(p)$ be a random bipartite graph with a constant $p \in(0,1)$. Then

$$
E E\left(G_{n_{1}, n_{2}}(p)\right)=e^{\frac{n}{2} p}\left(e^{O(\sqrt{n})}+o(1)\right) \quad \text { a.s. }
$$

if and only if $\lim _{n \rightarrow \infty} n_{2} / n_{1}=1$.

In this paper, by means of the symmetry in $G_{n_{1}, n_{2}}(p)$ and the spectral distribution of random matrix, we obtain lower and upper bounds for $E E\left(G_{n_{1}, n_{2}}(p)\right)$. For ease of analysis, we assume that $\lim _{n \rightarrow \infty} n_{2} / n_{1}:=y \in(0,1]$. We establish the estimate

\section{Theorem 1.}

$$
e^{n_{2} p}\left(e^{O(\sqrt{n})}+o(1)\right) \leq E E\left(G_{n_{1}, n_{2}}(p)\right) \leq e^{n_{1} p}\left(e^{O(\sqrt{n})}+O(1)\right) \quad \text { a.s. }
$$

Thus a weak version of Conjecture 1 follows readily:

$$
E E\left(G_{n_{1}, n_{2}}(p)\right)=e^{\frac{n}{2} p}\left(e^{O(\sqrt{n})}+O(1)\right) \quad \text { a.s. }
$$


holds, provided $\lim _{n \rightarrow \infty} n_{2} / n_{1}=1$ (i.e., $y=1$ ).

\section{Examples}

In this section, we give some analytical and numerical examples to demonstrate that our estimate (3) is valid, and is better than some existing bounds obtained by algebraic approaches.

Example 1. Let $G$ be a bipartite graph with vertex set $V=V_{1} \cup V_{2},|V|=\left|V_{1}\right|+\left|V_{2}\right|=n_{1}+n_{2}=n$ and $m$ edges. In [11] and [18], it was proved that

$$
\sqrt{n^{2}+4 m} \leq E E(G) \leq n-2+2 \cosh (\sqrt{m})
$$

where the equality on the left-hand side of (4) holds if and only if $G$ is an empty graph, while the equality on the right-hand side of (4) holds if and only if $G$ is a union of a complete bipartite graph and some isolated vertices. If $G \in \mathcal{G}_{n_{1}, n_{2}}(p)$, then the number of edges is $m=n_{1} n_{2} p$ a.s. [19]. Hence, the lower bounds in (4) becomes

$$
\sqrt{n^{2}+4 m}=\sqrt{n^{2}+4 n_{1} n_{2} p} \ll e^{n_{2} p}\left(e^{O(\sqrt{n})}+o(1)\right) \quad \text { a.s. }
$$

The upper bound in (4) becomes

$$
\begin{aligned}
n-2+2 \cosh (\sqrt{m}) & =n-2+e^{\sqrt{n_{1} n_{2} p}}+e^{-\sqrt{n_{1} n_{2} p}}=e^{n_{1} p}\left(e^{n_{1}(\sqrt{y p}-p)}+o(1)\right) \\
& =e^{n_{1} p} \cdot\left(e^{n\left(\frac{\sqrt{y p}-p}{1+y}\right)}+o(1)\right) \\
& \gg e^{n_{1} p}\left(e^{O(\sqrt{n})}+O(1)\right), \quad \text { a.s. }
\end{aligned}
$$

if $n_{2} / n_{1} \rightarrow y>p$.

Example 2. In [15], the authors bounded the Estrada index of a graph $G$ of order $n$ by its graph energy $E(G)$ :

$$
E E(G) \leq n-1+e^{\frac{E(G)}{2}},
$$

with equality if and only if $E(G)=0$. If $G \in \mathcal{G}_{n_{1}, n_{2}}(p)$, it can be shown that [24] $E(G)=$ $\left(2 n_{2} \sqrt{n_{1}} C(p) n^{-3 / 2}+o(1)\right) n^{3 / 2}$ a.s., where $C(p)>0$ is a constant depending on $p$. Hence, we have

$$
E E(G) \leq n-1+e^{\left(2 n_{2} \sqrt{n_{1}} C(p) n^{-\frac{3}{2}}+o(1)\right) n^{\frac{3}{2}}}, \quad \text { a.s. }
$$

We calculate the upper bound in (5) as

$$
\begin{aligned}
& n-1+e^{\left(2 n_{2} \sqrt{n_{1}} C(p) n^{-\frac{3}{2}}+o(1)\right) n^{\frac{3}{2}}} \\
= & e^{n_{1} p} \cdot\left(e^{\left(2 y C(p) n_{1}^{\frac{3}{2}} n^{-\frac{3}{2}}+o(1)\right) n^{\frac{3}{2}}-n_{1} p}+o(1)\right) \\
= & e^{n_{1} p} \cdot\left(e^{\left(2 y(1+y)^{-\frac{3}{2}} C(p)+o(1)\right) n^{\frac{3}{2}}-n p(1+y)^{-1}}+o(1)\right) \\
\gg & e^{n_{1} p}\left(e^{O(\sqrt{n})}+O(1)\right) . \quad \text { a.s. }
\end{aligned}
$$


Example 3. Let $G$ be a bipartite graph with $n$ vertices and $m \geq 1$ edges. Denote by $n_{0}$ the number of zero eigenvalues in the spectrum of $G$. It was proved in [25] and [12] that

$$
E E(G) \geq n_{0}+\left(n-n_{0}\right) \cosh \left(\sqrt{\frac{2 m}{n-n_{0}}}\right),
$$

where the equality holds if and only if $n-n_{0}$ is even, and $G$ consists of copies of complete bipartite graphs $K_{a_{i}, b_{i}}, i=1, \cdots,\left(n-n_{0}\right) / 2$, such that all products $a_{i} b_{i}$ are mutually equal. If $G \in \mathcal{G}_{n_{1}, n_{2}}(p)$, then the number of edges is $m=n_{1} n_{2} p$ a.s. Hence, the lower bound in (6) becomes

$$
\begin{aligned}
& n_{0}+\left(n-n_{0}\right) \cosh \left(\sqrt{\frac{2 m}{n-n_{0}}}\right)=n_{0}+\frac{1}{2}\left(n-n_{0}\right)\left(e^{\sqrt{\frac{2 n_{1} n_{2} p}{n-n_{0}}}}+e^{-\sqrt{\frac{2 n_{1} n_{2} p}{n-n_{0}}}}\right) \\
& \leq e^{n_{2} p}\left(\frac{n}{2} e^{\sqrt{\frac{2 n_{1} n_{2} p}{n}}-n_{2} p}+o(1)\right) \\
& =e^{n_{2} p}\left(\frac{n}{2} e^{\frac{\sqrt{2 y n p}-y n p}{1+y}}+o(1)\right) \\
& \ll e^{n_{2} p}\left(e^{O(\sqrt{n})}+o(1)\right) \text {. a.s. }
\end{aligned}
$$

Example 4. An upper bound for $E E\left(G_{n_{1}, n_{2}}(p)\right)$ was derived in [20] as

$$
E E\left(G_{n_{1}, n_{2}}(p)\right) \leq e^{n p}\left(e^{O(\sqrt{n})}+o(1)\right) \quad \text { a.s. }
$$

Since

$$
\frac{e^{n_{1} p}\left(e^{O(\sqrt{n})}+O(1)\right)}{e^{n p}\left(e^{O(\sqrt{n})}+o(1)\right)}=(1+o(1)) e^{-\frac{y n p}{1+y}}=o(1),
$$

our upper bound in Theorem 1 is better than that in (7).

In Table 1, we compare the estimates for $E E\left(G_{n_{1}, n_{2}}(1 / 2)\right)$ in Theorem 1 with numerical value obtained by Matlab software. The results are in good agreement with the theory.

Table 1. Estrada index of $G_{n_{1}, n_{2}}(p)$ with $n_{1}=3000$ and $p=1 / 2$. Numerical results are based on average over 10 independent runs.

\begin{tabular}{ccccc}
\hline $\mathbf{n}_{\mathbf{2}}$ & $\mathbf{y}$ & Theoretical lower bound & Numerical value & Theoretical upper bound \\
\hline 3000 & 1 & $e^{1500}\left(e^{O(77.46)}+o(1)\right)$ & $e^{1543.72}$ & $e^{1500}\left(e^{O(77.46)}+O(1)\right)$ \\
2700 & 0.9 & $e^{1350}\left(e^{O(75.50)}+o(1)\right)$ & $e^{1528.29}$ & $e^{1500}\left(e^{O(75.50)}+O(1)\right)$ \\
2400 & 0.8 & $e^{1200}\left(e^{O(73.48)}+o(1)\right)$ & $e^{1497.03}$ & $e^{1500}\left(e^{O(73.48)}+O(1)\right)$ \\
2100 & 0.7 & $e^{1050}\left(e^{O(71.41)}+o(1)\right)$ & $e^{1456.85}$ & $e^{1500}\left(e^{O(71.41)}+O(1)\right)$ \\
\hline
\end{tabular}

\section{Proof of Theorem 1}

This section is devoted to the proof of Theorem 1, which heavily relies on the symmetry in $G_{n_{1}, n_{2}}(p)$. Throughout the paper, we shall understand $p \in(0,1)$ as a constant. Let $M=\left(m_{i j}\right) \in \mathbb{R}^{n \times n}$ be a random matrix, where the entries $m_{i j}(i \leq j)$ are independent and identically distributed with 
$m_{i j}=m_{j i}$. We denote by the eigenvalues of $M$ by $\lambda_{1}(M), \lambda_{2}(M), \cdots, \lambda_{n}(M)$ and its empirical spectral distribution by

$$
\begin{aligned}
\Phi_{M}(x) & =\frac{1}{n} \cdot \#\left\{\lambda_{i}(M) \mid \lambda_{i}(M) \leq x, i=1,2, \cdots, n\right\} \\
& =\frac{1}{n} \sum_{i=1}^{n} 1_{\left\{\lambda_{i}(M) \leq x\right\}}
\end{aligned}
$$

Lemma 1. (Marčenko-Pastur Law [26]) Let $X=\left(x_{i j}\right) \in \mathbb{R}^{n_{2} \times n_{1}}$ be a random matrix, where the entries $x_{i j}$ are independent and identically distributed with mean zero and variance $p(1-p)$. Suppose that $n_{1}, n_{2}$ are functions of $n$, and $\lim _{n \rightarrow \infty} n_{2} / n_{1}=y \in(0, \infty)$. Then, with probability 1 , the empirical spectral distribution $\Phi_{\frac{1}{n_{1}} X X^{T}}(x)$ converges weakly to the Marčenko-Pastur Law $F_{y}$ as $n \rightarrow \infty$, where $F_{y}$ has the density

$$
f_{y}(x)=\frac{1}{2 \pi p(1-p) x y} \sqrt{(b-x)(x-a)} 1_{\{a \leq x \leq b\}}
$$

and has a point mass $1-1 / y$ at the origin if $y>1$, where $a=p(1-p)(1-\sqrt{y})^{2}$ and $b=p(1-p)(1+\sqrt{y})^{2}$.

The above result formulates the limit spectral distribution of $\frac{1}{n_{1}} X X^{T}$, which will be a key ingredient of our later derivation for $E E\left(G_{n_{1}, n_{2}}(p)\right)$. The main approach employed to prove the assertion is called moment approach. It can be shown that for each $k \in \mathbb{N}$,

$$
\lim _{n \rightarrow \infty} \int_{0}^{\infty} x^{k} d \Phi_{\frac{1}{n_{1}} X X^{T}}(x)=\int_{0}^{\infty} x^{k} d F_{y}(x) \quad \text { a.s. }
$$

We refer the reader to the seminal survey by Bai [26] for further details on the moment approach and the Marčenko-Pastur Law-like results.

The following two lemmas will be needed.

Lemma 2. (In Page 219 [27]), Let $\mu$ be a measure. Suppose that functions $g_{n}, h_{n}, f_{n}$ converge almost everywhere to functions $g, h, f$, respectively, and that $g_{n} \leq f_{n} \leq h_{n}$ almost everywhere. If $\int g_{n} d \mu \rightarrow$ $\int g d \mu$ and $\int h_{n} d \mu \rightarrow \int h d \mu$, then $\int f_{n} d \mu \rightarrow \int f d \mu$.

Lemma 3. (Weyl's inequality [28]) Let $X \in \mathbb{R}^{n \times n}, Y \in \mathbb{R}^{n \times n}$ and $Z \in \mathbb{R}^{n \times n}$ be symmetric matrices such that $X=Y+Z$. Suppose their eigenvalues are ordered as $\lambda_{1}(X) \geq \lambda_{2}(X) \geq \cdots \geq \lambda_{n}(X)$, $\lambda_{1}(Y) \geq \lambda_{2}(Y) \geq \cdots \geq \lambda_{n}(Y)$, and $\lambda_{1}(Z) \geq \lambda_{2}(Z) \geq \cdots \geq \lambda_{n}(Z)$, respectively. Then

$$
\lambda_{i}(Y)+\lambda_{n}(Z) \leq \lambda_{i}(X) \leq \lambda_{i}(Y)+\lambda_{1}(Z)
$$

for any $1 \leq i \leq n$.

Recall that the random bipartite graph $\mathcal{G}_{n_{1}, n_{2}}(p)$ consists of all bipartite graphs with vertex set $V=V_{1} \cup V_{2}$, in which the edges connecting vertices between $V_{1}$ and $V_{2}$ are chosen independently with probability $p \in(0,1)$. We assume $\left|V_{i}\right|=n_{i}(i=1,2), n_{1}+n_{2}=n$ and $\lim _{n \rightarrow \infty} n_{2} / n_{1}:=y \in(0,1]$.

For brevity, let $A_{n}=A\left(G_{n_{1}, n_{2}}(p)\right)$ be the adjacency matrix, and denote by $Q_{n}=\left(q_{i j}\right) \in \mathbb{R}^{n \times n}$ a quasi-unit matrix, where $q_{i j}=q_{j i}=1$ if $i, j \in V_{1}$ or $i, j \in V_{2}$, and $q_{i j}=q_{j i}=0$ otherwise. Let 
$I_{n} \in \mathbb{R}^{n \times n}$ be the unit matrix and $J_{n} \in \mathbb{R}^{n \times n}$ be the matrix whose all entries are equal to 1 . By labeling the vertices appropriately, we obtain

$$
\bar{A}_{n}:=A_{n}-p\left(J_{n}-Q_{n}\right)=\left(\begin{array}{cc}
0 & X^{T} \\
X & 0
\end{array}\right) \in \mathbb{R}^{n \times n},
$$

where $X=\left(x_{i j}\right) \in \mathbb{R}^{n_{2} \times n_{1}}$ is a random matrix with all entries $x_{i j}$ being independent and identically distributed with mean zero and variance $p(1-p)$. For $\lambda \in \mathbb{R}$, by basic matrix transforms, namely, taking the determinants of both sides of

$$
\left(\begin{array}{cc}
\lambda I_{n_{1}} & 0 \\
-X & \lambda I_{n_{2}}
\end{array}\right)\left(\begin{array}{cc}
\lambda I_{n_{1}} & -X^{T} \\
0 & \lambda I_{n_{2}}-\lambda^{-1} X X^{T}
\end{array}\right)=\lambda\left(\begin{array}{cc}
\lambda I_{n_{1}} & -X^{T} \\
-X & \lambda I_{n_{2}}
\end{array}\right)
$$

we have

$$
\lambda^{n_{1}} \operatorname{det}\left(\lambda^{2} I_{n_{2}}-X X^{T}\right)=\lambda^{n_{2}} \operatorname{det}\left(\lambda I_{n}-\bar{A}_{n}\right),
$$

where $\operatorname{det}(M)$ is the determinant of matrix $M$. Consequently,

$$
\lambda^{n_{1}} n_{1}^{n_{2}} \operatorname{det}\left(\frac{\lambda^{2}}{n_{1}} I_{n_{2}}-\frac{1}{n_{1}} X X^{T}\right)=\left(\sqrt{n_{1}}\right)^{n} \lambda^{n_{2}} \operatorname{det}\left(\frac{\lambda}{\sqrt{n_{1}}} I_{n}-\frac{\bar{A}_{n}}{\sqrt{n_{1}}}\right) .
$$

Therefore, the eigenvalues of $\bar{A}_{n} / \sqrt{n_{1}}$ are symmetric: If $\bar{\lambda} \neq 0$, then $\bar{\lambda}$ is the eigenvalue of $\bar{A}_{n} / \sqrt{n_{1}}$ if and only if $\bar{\lambda}^{2}$ is the eigenvalue of $X X^{T} / n_{1}$. Since $X X^{T}$ is positive semi-definite, we know that $\bar{A}_{n} / \sqrt{n_{1}}$ has at least $n_{1}-n_{2}$ zero eigenvalues and its spectrum can be arranged in a non-increasing order as

$$
\bar{\lambda}_{1} \geq \cdots \geq \bar{\lambda}_{n_{2}} \geq \underbrace{0=\cdots=0}_{n_{1}-n_{2}} \geq \begin{gathered}
\bar{\lambda}_{n_{1}+1} \geq \cdots \geq \begin{array}{c}
\bar{\lambda}_{n} \\
-\bar{\lambda}_{n_{2}} \\
\| \bar{\lambda}_{1}
\end{array} \\
-\bar{\lambda}_{1}
\end{gathered},
$$

assuming $n_{1} \geq n_{2}$ when $n$ is large enough.

In what follows, we shall investigate $E E\left(G_{n_{1}, n_{2}}(p)\right)$ and prove Theorem 1 through a series of propositions. For convenience, we sometimes write $E E(M):=\sum_{i=1}^{n} e^{\lambda_{i}(M)}$ for a real symmetric matrix $M \in \mathbb{R}^{n \times n}$. Thus, $E E\left(G_{n_{1}, n_{2}}(p)\right)=E E\left(A_{n}\right)$.

\section{Proposition 1.}

$$
\lim _{n \rightarrow \infty} \int_{1}^{\infty} e^{x} d \Phi_{\frac{1}{n_{1}} X X^{T}}(x)=\int_{1}^{\infty} e^{x} d F_{y}(x) \quad \text { a.s. }
$$

Proof. Let $\phi_{\frac{1}{n_{1}} X X^{T}}$ be the density of $\Phi_{\frac{1}{n_{1}} X X^{T}}$. By means of Lemma 1, we get $\phi_{\frac{1}{n_{1}} X X^{T}}$ converges to $f_{y}$ a.s. as $n$ tends to infinity. It follows from the bounded convergence theorem that

$$
\lim _{n \rightarrow \infty} \int_{0}^{1} e^{x} d \Phi_{\frac{1}{n_{1}} X X^{T}}(x)=\int_{0}^{1} e^{x} d F_{y}(x) \quad \text { a.s. }
$$

By Lemma 1 we know that there exists a large $\omega<\infty$ such that all eigenvalues of $\frac{1}{n_{1}} X X^{T}$ do not exceed $\omega$. Since the expansion $e^{x}=\sum_{k=0}^{\infty} x^{k} / k$ ! converges uniformly on $[0, \omega]$, we obtain from (8) that

$$
\lim _{n \rightarrow \infty} \int_{0}^{\infty} e^{x} d \Phi_{\frac{1}{n_{1}} X X^{T}}(x)=\int_{0}^{\omega} e^{x} d F_{y}(x)=\int_{0}^{\infty} e^{x} d F_{y}(x) \quad \text { a.s. }
$$


Combining (10) with (11) we derive

$$
\begin{aligned}
\lim _{n \rightarrow \infty} \int_{1}^{\infty} e^{x} d \Phi_{\frac{1}{n_{1}} X X^{T}}(x) & =\lim _{n \rightarrow \infty}\left(\int_{0}^{\infty} e^{x} d \Phi_{\frac{1}{n_{1}} X X^{T}}(x)-\int_{0}^{1} e^{x} d \Phi_{\frac{1}{n_{1}} X X^{T}}(x)\right) \\
& =\int_{0}^{\infty} e^{x} d F_{y}(x)-\int_{0}^{1} e^{x} d F_{y}(x) \\
& =\int_{1}^{\infty} e^{x} d F_{y}(x) \text { a.s. }
\end{aligned}
$$

\section{Proposition 2.}

$$
E E\left(\frac{\bar{A}_{n}}{\sqrt{n_{1}}}\right)=n_{1}-n_{2}+n_{2}\left(\frac{\int_{\sqrt{a}}^{\sqrt{b}} 2 \cosh (x) \sqrt{\left(\frac{b}{x^{2}}-1\right)\left(1-\frac{a}{x^{2}}\right)} d x}{\pi p(1-p) y}+o(1)\right) \quad \text { a.s. }
$$

where $a$ and $b$ are given as in Lemma 1.

Proof. Define

$$
\Psi(x)=\frac{1}{n_{2}} \sum_{i=1}^{n_{2}} 1_{\left\{\sqrt{\lambda_{i}\left(\frac{1}{n_{1}} X X^{T}\right)} \leq x\right\}} .
$$

Then we have

$$
e^{\bar{\lambda}_{1}}+\cdots+e^{\bar{\lambda}_{n_{2}}}=n_{2} \int_{0}^{\infty} e^{x} d \Psi(x)=n_{2} \int_{0}^{\infty} e^{\sqrt{x}} d \Phi_{\frac{1}{n_{1}} X X^{T}}(x)
$$

Analogous to the proof of (10) we derive

$$
\lim _{n \rightarrow \infty} \int_{0}^{1} e^{\sqrt{x}} d \Phi_{\frac{1}{n_{1}} X X^{T}}(x)=\int_{0}^{1} e^{\sqrt{x}} d F_{y}(x) \quad \text { a.s. }
$$

For any $x \in[1, \infty)$, we have $0 \leq e^{\sqrt{x}} \leq e^{x}$. By Lemma 2 and Proposition 1 we deduce that

$$
\lim _{n \rightarrow \infty} \int_{1}^{\infty} e^{\sqrt{x}} d \Phi_{\frac{1}{n_{1}} X X^{T}}(x)=\int_{1}^{\infty} e^{\sqrt{x}} d F_{y}(x) \text { a.s. }
$$

Accordingly, we have

$$
\lim _{n \rightarrow \infty} \int_{0}^{\infty} e^{\sqrt{x}} d \Phi_{\frac{1}{n_{1}} X X^{T}}(x)=\int_{0}^{\infty} e^{\sqrt{x}} d F_{y}(x) \text { a.s. }
$$

It follows from (12) and (13) that

$$
\begin{aligned}
& e^{\bar{\lambda}_{1}}+\cdots+e^{\bar{\lambda}_{n_{2}}} \\
\rightarrow & n_{2} \int_{0}^{\infty} e^{\sqrt{x}} d F_{y}(x) \\
= & \int_{a}^{b} \frac{e^{\sqrt{x}}}{2 \pi p(1-p) x y} \sqrt{(b-x)(x-a)} d x \\
= & \frac{1}{\pi p(1-p) y} \int_{\sqrt{a}}^{\sqrt{b}} e^{x} \sqrt{\left(\frac{b}{x^{2}}-1\right)\left(1-\frac{a}{x^{2}}\right)} d x \quad \text { a.s. }
\end{aligned}
$$


Next, we calculate the sum of the exponentials of the smallest $n_{2}$ eigenvalues of $\bar{A}_{n} / \sqrt{n_{1}}$. Similarly as in (12), we obtain

$$
e^{\bar{\lambda}_{n_{1}+1}}+\cdots+e^{\bar{\lambda}_{n}}=n_{2} \int_{0}^{\infty} e^{-x} d \Psi(x)=n_{2} \int_{0}^{\infty} e^{-\sqrt{x}} d \Phi_{\frac{1}{n_{1}} X X^{T}}(x) .
$$

Noting that $0 \leq e^{-\sqrt{x}} \leq e^{x}$ for $x \in[1, \infty)$, we likewise have

$$
\lim _{n \rightarrow \infty} \int_{0}^{\infty} e^{-\sqrt{x}} d \Phi_{\frac{1}{n_{1}} X X^{T}}(x)=\int_{0}^{\infty} e^{-\sqrt{x}} d F_{y}(x) \quad \text { a.s. }
$$

by employing Lemma 2 and Proposition 1. It then follows from (15) and (16) that

$$
\begin{aligned}
& e^{\bar{\lambda}_{n_{1}+1}}+\cdots+e^{\bar{\lambda}_{n}} \\
\rightarrow & n_{2} \int_{0}^{\infty} e^{-\sqrt{x}} d F_{y}(x) \\
= & \frac{1}{\pi p(1-p) y} \int_{\sqrt{a}}^{\sqrt{b}} e^{-x} \sqrt{\left(\frac{b}{x^{2}}-1\right)\left(1-\frac{a}{x^{2}}\right)} d x \quad \text { a.s. }
\end{aligned}
$$

Finally, combining (14), (17) and the fact that $\bar{\lambda}_{n_{2}+1}=\cdots=\bar{\lambda}_{n_{1}}=0$, we readily deduce the assertion of Proposition 2.

\section{Proposition 3.}

$$
\begin{aligned}
& n_{1}-n_{2}+n_{2}\left(\frac{\left(e^{-\sqrt{n_{1} b}}+e^{\sqrt{n_{1} a}}\right) C}{\pi p(1-p) y}+o(1)\right) \\
& \quad \leq E E\left(\bar{A}_{n}\right) \leq n_{1}-n_{2}+n_{2}\left(\frac{\left(e^{-\sqrt{n_{1} a}}+e^{\sqrt{n_{1} b}}\right) C}{\pi p(1-p) y}+o(1)\right) \quad \text { a.s., }
\end{aligned}
$$

where $a, b$ are given as in Lemma 1 , and $C=\int_{\sqrt{a}}^{\sqrt{b}} \sqrt{\left(\frac{b}{x^{2}}-1\right)\left(1-\frac{a}{x^{2}}\right)} d x$.

Proof. Define

$$
\tilde{\Psi}(x)=\frac{1}{n_{2}} \sum_{i=1}^{n_{2}} 1_{\left\{\sqrt{n_{1} \lambda_{i}\left(\frac{1}{n_{1}} X X^{T}\right)} \leq x\right\}} .
$$

Then we have $\tilde{\Psi}(x)=\Psi\left(x / \sqrt{n_{1}}\right)$. Therefore, the sum of the exponentials of the largest $n_{2}$ eigenvalues of $\bar{A}_{n}$ is

$$
\begin{aligned}
e^{\lambda_{1}\left(\bar{A}_{n}\right)}+\cdots+e^{\lambda_{n_{2}}\left(\bar{A}_{n}\right)} & =n_{2} \int_{0}^{\infty} e^{x} d \tilde{\Psi}(x)=n_{2} \int_{0}^{\infty} e^{\sqrt{n_{1}} x} d \Psi(x) \\
& =n_{2} \int_{0}^{\infty} e^{\sqrt{n_{1} x}} d \Phi_{\frac{1}{n_{1}} X X^{T}}(x)
\end{aligned}
$$

Analogous to the proof of (14) we obtain

$$
\begin{aligned}
& \lim _{n \rightarrow \infty} e^{\lambda_{1}\left(\bar{A}_{n}\right)}+\cdots+e^{\lambda_{n_{2}}\left(\bar{A}_{n}\right)} \\
= & \frac{1}{\pi p(1-p) y} \int_{\sqrt{a}}^{\sqrt{b}} e^{\sqrt{n_{1}} x} \sqrt{\left(\frac{b}{x^{2}}-1\right)\left(1-\frac{a}{x^{2}}\right)} d x \\
\in & {\left[\frac{C e^{\sqrt{n_{1} a}}}{\pi p(1-p) y}, \frac{C e^{\sqrt{n_{1} b}}}{\pi p(1-p) y}\right] \quad \text { a.s. } }
\end{aligned}
$$


where $C=\int_{\sqrt{a}}^{\sqrt{b}} \sqrt{\left(\frac{b}{x^{2}}-1\right)\left(1-\frac{a}{x^{2}}\right)} d x$. Similarly, the sum of the exponentials of the smallest $n_{2}$ eigenvalues of $\bar{A}_{n}$ satisfies

$$
\begin{aligned}
& \lim _{n \rightarrow \infty} e^{\lambda_{n_{1}+1}\left(\bar{A}_{n}\right)}+\cdots+e^{\lambda_{n}\left(\bar{A}_{n}\right)} \\
= & \frac{1}{\pi p(1-p) y} \int_{\sqrt{a}}^{\sqrt{b}} e^{-\sqrt{n_{1}} x} \sqrt{\left(\frac{b}{x^{2}}-1\right)\left(1-\frac{a}{x^{2}}\right)} d x \\
\in & {\left[\frac{C e^{-\sqrt{n_{1} b}}}{\pi p(1-p) y}, \frac{C e^{-\sqrt{n_{1} a}}}{\pi p(1-p) y}\right] \quad \text { a.s. } }
\end{aligned}
$$

Combining (18), (19) and the fact that $\lambda_{n_{2}+1}\left(\bar{A}_{n}\right)=\cdots=\lambda_{n_{1}}\left(\bar{A}_{n}\right)=0$, we complete the proof of Proposition 3.

\section{Proposition 4.}

$$
e^{-p n_{1}}\left(e^{O\left(\sqrt{n_{1}}\right)}+O(1)\right) \leq E E\left(A_{n}\right) \leq e^{p n_{1}}\left(e^{O\left(\sqrt{n_{1}}\right)}+O(1)\right) \quad \text { a.s. }
$$

Proof. Since $n_{1} \geq n_{2}$ for large $n$, by the Geršhgorin circle theorem we deduce

$$
-p n_{1} \leq \lambda_{n}\left(p\left(J_{n}-Q_{n}\right)\right) \leq \cdots \leq \lambda_{1}\left(p\left(J_{n}-Q_{n}\right)\right) \leq p n_{1}
$$

In view of Lemma 3 and $A_{n}=\bar{A}_{n}+p\left(J_{n}-Q_{n}\right)$, we get

$$
\lambda_{i}\left(\bar{A}_{n}\right)+\lambda_{n}\left(p\left(J_{n}-Q_{n}\right)\right) \leq \lambda_{i}\left(A_{n}\right) \leq \lambda_{i}\left(\bar{A}_{n}\right)+\lambda_{1}\left(p\left(J_{n}-Q_{n}\right)\right)
$$

for all $i$. Consequently, $e^{-p n_{1}} E E\left(\bar{A}_{n}\right) \leq E E\left(A_{n}\right) \leq e^{p n_{1}} E E\left(\bar{A}_{n}\right)$. It then follows from Proposition 3 that

$$
\begin{aligned}
e^{-p n_{1}}\left(n_{1}-n_{2}\right)+ & e^{-p n_{1}} n_{2}\left(\frac{\left(e^{-\sqrt{n_{1} b}}+e^{\sqrt{n_{1} a}}\right) C}{\pi p(1-p) y}+o(1)\right) \\
& \leq E E\left(A_{n}\right) \leq e^{p n_{1}}\left(n_{1}-n_{2}\right)+e^{p n_{1}} n_{2}\left(\frac{\left(e^{-\sqrt{n_{1} a}}+e^{\sqrt{n_{1} b}}\right) C}{\pi p(1-p) y}+o(1)\right) \quad \text { a.s. }
\end{aligned}
$$

Note that the rate of convergence in (18) as well as (19) can be bounded by $O\left(n^{-1}\right)$ using the moment approach (for instance, see Theorem 4.5.5 in [29] ) and the estimates in [26] (pp. 621-623). Hence, the infinitesimal quantity $o(1)$ on both sides of (20) is equivalent to $O\left(n^{-1}\right)$. Inserting $n_{1}=n /(1+y)$ and $n_{2}=y n_{1}$ into (20), we have

$$
e^{-p n_{1}}\left(e^{O\left(\sqrt{n_{1}}\right)}+O(1)\right) \leq E E\left(A_{n}\right) \leq e^{p n_{1}}\left(e^{O\left(\sqrt{n_{1}}\right)}+O(1)\right) \quad \text { a.s. }
$$

as desired.

With Proposition 4 in our hands, we quickly get the proof of our main result.

Proof of Theorem 1. Since $n_{1}=O(n)$, the upper bound in Proposition 4 yields $E E\left(G_{n_{1}, n_{2}}(p)\right) \leq$ $e^{n_{1} p}\left(e^{O(\sqrt{n})}+O(1)\right)$ a.s. On the other hand, the lower bound $E E\left(G_{n_{1}, n_{2}}(p)\right) \geq e^{n_{2} p}\left(e^{O(\sqrt{n})}+o(1)\right)$ a.s. can be easily read out from Theorem 3.1 of [20]. 
We mention that it is possible that our method can be pushed further to obtain sharper bounds, for example, a more fine-grained analysis in Proposition 2 could give better estimates for the second order terms in the expansion for $\ln \left(E E\left(A_{n}\right)\right)$.

\section{Acknowledgments}

The author would like to thank the anonymous reviewers and the editor for their helpful and constructive comments that have improved the presentation of the manuscript. This work is funded by the National Natural Science Foundation of China (11505127), the Shanghai Pujiang Program (15PJ1408300), and the Program for Young Excellent Talents in Tongji University (2014KJ036).

\section{Conflicts of Interest}

The author declares no conflict of interest.

\section{References}

1. Garlaschelli, D.; Ruzzenenti, F.; Basosi, R. Complex networks and symmetry I: A review. Symmetry 2010, 2, 1683-1709.

2. Cvetković, D.M.; Doob, M.; Gutman, I.; Torgašev, A. Recent Results in the Theory of Graph Spectra; North-Holland: Amsterdam, The Netherlands, 1988.

3. Estrada, E. Characterization of 3D molecular structure. Chem. Phys. Lett. 2000, 319, 713-718.

4. Estrada, E. Characterization of the amino acid contribution to the folding degree of proteins. Proteins 2004, 54, 727-737.

5. Ginosar, Y.; Gutman, I.; Mansour, T.; Schork, M. Estrada index and Chbeyshev polynomials. Chem. Phys. Lett. 2008, 454, 145-147.

6. Garrido, A. Classifying entropy measures. Symmetry 2011, 3, 487-502.

7. Dehmer, M. Information theory of networks. Symmetry 2010, 2, 767-779.

8. Estrada, E.; Rodríguez-Velázquez, J.A. Subgraph centrality in complex networks. Phys. Rev. E 2005, 71, doi:10.1103/PhysRevE.71.056103.

9. Shang, Y. Biased edge failure in scale-free networks based on natural connectivity. Indian J. Phys. 2012, 86, 485-488.

10. Shang, Y. Random lifts of graphs: Network robustness based on the Estrada index. Appl. Math. E-Notes 2012, 12, 53-61.

11. De La Peña, J.A.; Gutman, I.; Rada, J. Estimating the Estrada index. Lin. Algebra Appl. 2007, 427, 70-76.

12. Gutman, I. Lower bounds for Estrada index. Publ. Inst. Math. (Beograd) 2008, 83, 1-7.

13. Gutman, I.; Deng, H.; Radenković, S. The Estrada index: An updated survey. In Selected Topics on Applications of Graph Spectra; Cvetković, D.M., Gutman, I., Eds.; Matematiki Institut SANU: Beograd, Serbia, 2011; pp. 155-174.

14. Shang, Y. Lower bounds for the Estrada index of graphs. Electron. J. Linear Algebra 2012, 23, $664-668$. 
15. Liu, J.; Liu, B. Bounds of the Estrada index of graphs. Appl. Math. J. Chin. Univ. 2010, 25, 325-330.

16. Shang, Y. Lower bounds for the Estrada index using mixing time and Laplacian spectrum. Rocky Mt. J. Math. 2013, 43, 2009-2016.

17. Shang, Y. The Estrada index of evolving graphs. Appl. Math. Comput. 2015, 250, 415-423.

18. Zhao, H.; Jia, Y. On the Estrada index of bipartite graph. MATCH Commun. Math. Comput. Chem. 2009, 61, 495-501.

19. Janson, S.; Łuczak, T.; Ruciński, A. Random Graphs; Wiley: New York, NY, USA, 2000.

20. Chen, Z.; Fan, Y.; Du, W. Estrada index of random graphs. MATCH Commun. Math. Comput. Chem. 2012, 68, 825-834.

21. Schaerf, A. A survey of automated timetabling. Artif. Intell. Rev. 1999, 13, 87-127.

22. Shang, Y. Estimation of the shortest average distance in bipartite networks with given density. $J$. Phys. Soc. Jpn. 2011, 80, doi:10.1143/JPSJ.80.055001.

23. Moon, T.K. Error Correction Coding: Mathematical Methods and Algorithms; Wiley: New York, NY, USA, 2005.

24. Du, W.; Li, X.; Li, Y. The energy of random graphs. Linear Algebra Appl. 2011, 435, 2334-2346.

25. Gutman, I.; Radenković, S. A lower bound for the Estrada index of bipartite molecular graphs. Kragujev. J. Sci. 2007, 29, 67-72.

26. Bai, Z. Methodologies in spectral analysis of large dimensional random matrices, a review. Stat. Sin. 1999, 9, 611-677.

27. Billingsley, P. Probability and Measure, 3rd ed.; Wiley: New York, NY, USA, 1995.

28. Weyl, H. Das asymptotische Verteilungsgesetz der Eigenwerte linearer partieller Differentialgleichungen. Math. Ann. 1912, 71, 441-479.

29. Chung, K.L. A Course in Probability Theory; Academic Press: San Diego, CA, USA, 2001.

(C) 2015 by the author; licensee MDPI, Basel, Switzerland. This article is an open access article distributed under the terms and conditions of the Creative Commons Attribution license (http://creativecommons.org/licenses/by/4.0/). 